\title{
The population of W Ursae Majoris-type binaries in the solar neighborhood
}

\author{
K. Gazeas \\ National and Kapodistrian University of Athens, Department of Physics, \\ Section of Astrophysics, Astronomy and Mechanics, GR 15784 Zografos, \\ Athens, Greece, (E-mail: kgaze@phys.uoa.gr)
}

Received: October 31, 2019; Accepted: January 2, 2020

\begin{abstract}
W Ursae Majoris-type binaries belong to the old population of our Galaxy, while their metallicity is close to solar. Their physical properties, kinematics and spatial distribution reflect the properties of their stellar progenitors. This study focuses on the spatial distribution of W UMa's in our solar neighborhood within a $500 \mathrm{pc}$ radius, with a combined astrometric, photometric and spectroscopic determination of their stellar parameters. The sample is carefully selected, in order to fulfill certain criteria, and has well defined metallicity and distance parameters. H-R diagram, as well as similar correlation plots (mass-radius and mass-luminosity), show that the primary (more massive) components in such systems are located close or below the ZAMS region, while secondary components seem to be evolved, as a result of their common envelope geometry. Some prominent outliers are carefully examined in order to judge the environmental properties and evolution in certain locations of the Milky Way. It is found that metallicity is not correlated with distance, but there is a weak correlation between metallicity $[\mathrm{M} / \mathrm{H}]$ and evolution state, as it is expressed by the location of the systems in the H-R diagram, the type of binary (A or W), and temperature.
\end{abstract}

Key words: binaries: W UMa-type - stars: physical parameters - stellar evolution

\section{Introduction}

W Ursae Majoris-type binaries ${ }^{1}$ are frequently observed as field targets, as well as within multiple stellar populations, open and globular clusters. Their combined photometric and spectroscopic study is a key tool for absolute physical property determinations, such as mass, radius, temperature, and therefore luminosity. It is well known that W UMa binaries belong to the old population of our Galaxy and their metallicity is close to solar. Certain empirical relations, extracted from various correlation diagrams, show that their physical parameters can be predicted up to a certain level, which, in turn, can be used as a tool

\footnotetext{
${ }^{1} \mathrm{~W}$ UMa binaries are overcontact systems of sufficiently late spectral type to have convective outer envelopes.
} 
for distance indication. Various studies (Hilditch et al., 1988; Gazeas \& Niarchos, 2006; Gazeas \& Stẹpień, 2008; Gazeas, 2009; Michel et al., 2019) showed that this tool can be widely used, since it results from their evolution status. The metallicities have been recently studied in detailed work by Rucinski et al. (2013) who performed a thorough study of 90 targets and concluded that $\mathrm{W}$ UMas have roughly solar metallicity, with the majority (52 targets) showing: $0.32<(B-V)_{0}<0.62$ or $0.65<[M / H]_{1}<+0.50$ (trend adjusted). They also showed that their kinematic properties are close to solar, while they belong to the thin disk population (age between 3.5-5 Gyr), based on the F-spectral type systems.

\section{The current study}

This study focuses on the spatial distribution of W UMa binaries in our solar neighborhood within a $500 \mathrm{pc}$ radius, using combined astrometric, photometric and spectroscopic determination of their stellar parameters. The sample is carefully selected, in order to fulfill certain criteria and have well defined metallicity and distance parameters. H-R diagram, as well as similar correlation plots (Mass-Radius and Mass-Luminosity) show that the primary (more massive) components are located close or below the ZAMS, while secondary ones seem to be evolved, as a result of their common envelope geometry. Some outliers are still prominent and are carefully examined in order to judge environmental properties and evolution in certain locations of the Milky Way. These outliers are connected with either a "third light" parameter (they are members of multiple systems), or have low inclination (causing large uncertainties in physical parameters) and/or are magnetically active (resulting in light curve asymmetries).

\section{Results}

The $[\mathrm{M} / \mathrm{H}]$ distribution of $\mathrm{W}$ UMas in distance is shown in Fig. 1. The metallicities are concentrated on the solar value (zero). A few systems are prominently higher than the entire sample (AB And, SW Lac, MS Vir, V502 Oph, V523 Cas, and $\mathrm{V} 2357 \mathrm{Oph}$ ). These six systems are $\mathrm{W}$ UMa binaries of the W-subtype, i.e. the more massive component in the system is the cooler one. None of these prominent systems belong to the A-subtype, i.e. where the more massive component in the system is the hotter one. (MS Vir is not determined yet, but there is strong evidence for $\mathrm{W}$-subtype). The spatial distribution of W UMas is shown in Fig. 2 in equatorial coordinates. Three of the above outliers (AB And, SW Lac, and V523 Cas) are relatively close to each other on the sky (left panel in Fig. 2), while the other three (MS Vir, V502 Oph, and V2357 Oph) are in the opposite sky direction (right panel in Fig. 2). Examining the spatial distribution in Cartesian coordinates (Fig. 3), we see that all high metallicity systems ex- 


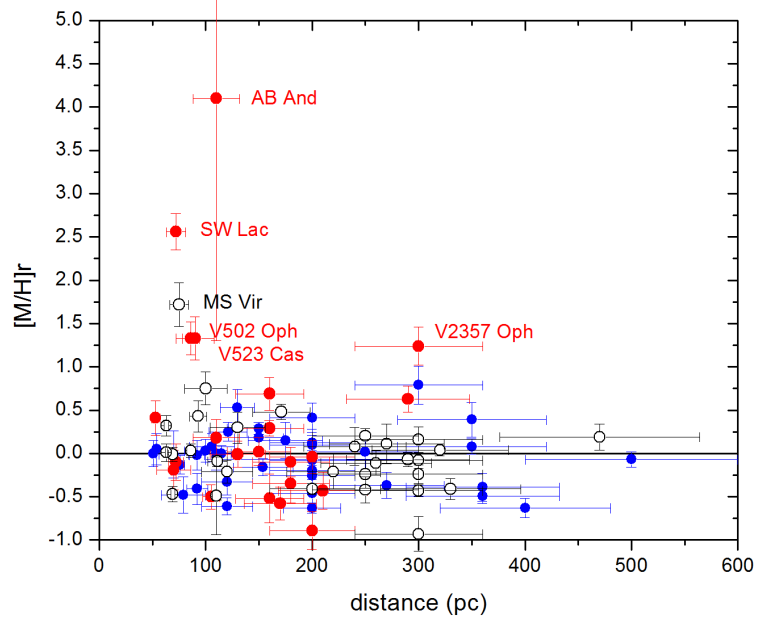

Figure 1. The majority of W UMa binaries have solar metallicity, although six outliers are prominent, with five being at a distance of $\approx 100 \mathrm{pc}$ (see text for details).
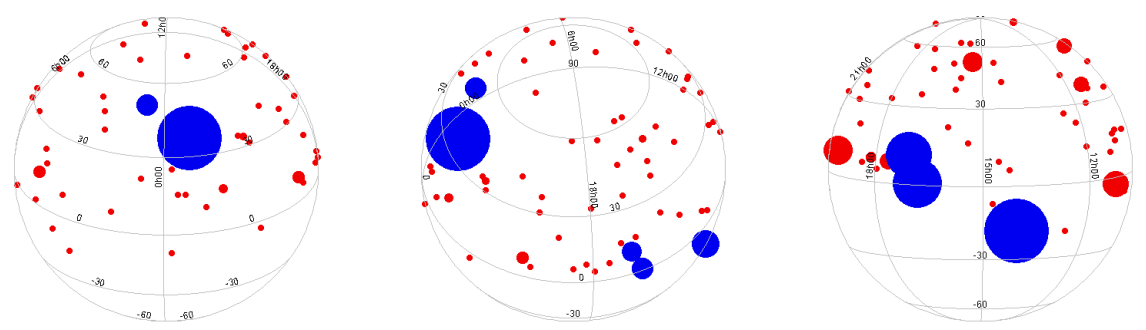

Figure 2. Spatial distribution of W UMa binaries in equatorial coordinates. High-metallicity systems (large blue circles) are located in two separate and opposite directions. Low-metallicity targets (small red circles) are spread towards all directions.

cept V2357 Oph are very close to the Sun, at distances of $\approx 100 \mathrm{pc}$. This is also prominent in Fig. 2, where the five systems are at a roughly the same distance, while V2357 Oph is much farther. This study has found that the metallicity is not correlated with distance (within $r<500 \mathrm{pc}$ ) but there is a weak correlation between metallicity $[\mathrm{M} / \mathrm{H}]$ and evolution state (expressed by the H-R diagram location, and A- or W-subtype). High metallicity systems are located in two almost opposite directions: towards the Andromeda-Cassiopeia-Lacerta region and towards the Ophiuchus-Libra-Virgo region. There is a weak connection with Galactic coordinates, since the six outliers are close to the Galactic plane. High metallicity systems have certain properties: they are very close to the solar neighborhood, clumped in certain directions and all are $\mathrm{W}$-subtype. 

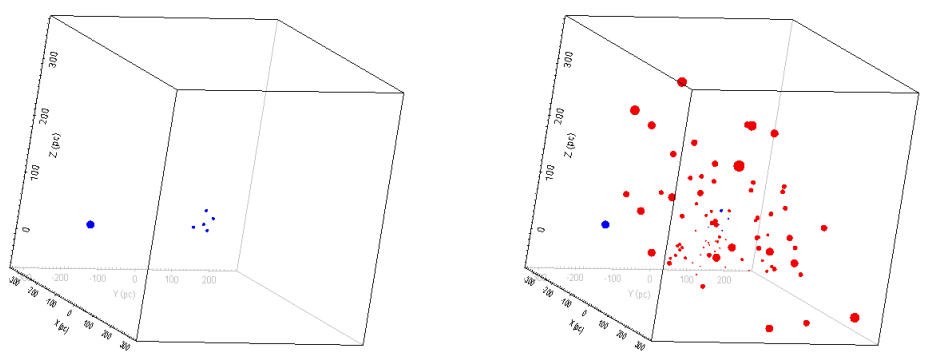

Figure 3. Spatial distribution of W UMa binaries in Cartesian coordinates. High-metallicity systems (left panel) are located close to our solar vicinity, while low-metallicity targets are covering all distance scales up to 500 pc.

Acknowledgements. The author is thankful to Prof. S. Rucinski for inspiring this study by various stimulating discussions and constructive comments. A large volume of the photometric data used in this study were collected in the frame of the photometric observations with the robotic and remotely controlled telescope at the University of Athens Observatory (UOAO; Gazeas, 2016).

\section{References}

Gazeas, K., The robotic and remotely controlled telescope at the University of Athens Observatory. in , Revista Mexicana de Astronomia y Astrofisica Conference Series, Vol. 48, 22-23

Gazeas, K. \& Stẹpień, K., Angular momentum and mass evolution of contact binaries. 2008, Mon. Not. R. Astron. Soc., 390, 1577, DOI: 10.1111/j.1365-2966.2008.13844.x

Gazeas, K. D., Physical parameters of contact binaries through 2-D and 3-D correlation diagrams. 2009, Communications in Asteroseismology, 159, 129

Gazeas, K. D. \& Niarchos, P. G., Masses and angular momenta of contact binary stars. 2006, Mon. Not. R. Astron. Soc., 370, L29, DOI: 10.1111/j.1745-3933.2006.00182.x

Hilditch, R. W., King, D. J., \& McFarlane, T. M., The evolutionary state of contact and near-contact binary stars. 1988, Mon. Not. R. Astron. Soc., 231, 341, DOI: $10.1093 / \mathrm{mnras} / 231.2 .341$

Michel, R., Acerbi, F., Barani, C., \& Martignoni, M., Multicolor Study of V1009 Per, a Close Binary System at the Beginning of the Overcontact Phase, and of CRTS J031642.2+332639, a New Binary System in the Same Field. 2019, Rev. Mex. Astron. Astrofis., 55, 65

Rucinski, S. M., Pribulla, T., \& Budaj, J., Spectroscopic Metallicity Determinations for W UMa-type Binary Stars. 2013, Astron. J., 146, 70, DOI: 10.1088/0004$6256 / 146 / 3 / 70$ 